\title{
Sobre tres tablas pintadas del siglo XVI
}

\author{
María Concepción García Gainza \\ Universidad de Navarra
}

\begin{abstract}
RESUMEN: Se dan a conocer tres tablas inéditas de pintura del siglo XVI que pertenecieron al museo del Colegio Capuchinos de Lecároz (Navarra). Representan la Lapidación de San Esteban, San Julián y Santa Basilisa haciendo la limosna y santo obispo bendiciendo los campos. Sus dimensiones aproximadamente iguales hacen pensar que las tres tablas formaron parte de un retablo. Tanto por su iconografía como por su colorido manierista pueden considerarse como pinturas propias de la Contrarreforma de hacia 1590, lo que se hace evidente en la Lapidación de San Esteban que copia una estampa grabada de Ecclesiae Militantes Triunphi (Roma, 1585) que recoge la iconografía jesuítica de la Contrarreforma dedicada a los primeros mártires del cristianismo a los que se relaciona con la doctrina teológica del Concilio de Trento. El grabado se debe a Giovanni Battista Cavallieri. El anónimo pintor de las tablas de Lecároz firma con el monograma F M, la F surmontada por la o y la M por la a o la $\mathrm{z}$.
\end{abstract}

PALABRAS CLAVE: Tablas pintadas, Siglo XVI, Iconografía jesuítica de la Contrarreforma, Giovanni Battista Cavallieri, Grabador.

\section{Sobre tres tablas pintadas del siglo XVI}

ABSTRACT: The objective of this article is to announce three unpublished painting panels of the XVI century which belonged to the museum of the Capuchin College of Lecároz (Navarre). They represent the Stoning of Saint Stephan, Saint Julian and Saint Basilisa giving money to beggars and a Saint Bishop blessing the fields. Their approximately equal dimensions make us think that the three panels were a part of the same altarpiece. Because of their iconography and manneristic coloring may be considered as paintings of the Counter-Reformation in about 1590, what becomes rather obvious in the Stoning of Saint Stephan which reproduces an engraved stamp of Ecclesiae Militantis Triunphi (Rome, 1585) collecting the Jesuit iconography of the Counter-Reformation dedicated to the early martyrs of Christianism, which are related to the theological of Council of Trento. The engraving is due to Giovanni Battista Cavallieri. The anonymous painter of the Lecároz panels signs them with the monogram F M, the «F» letter overtopped by an «o» and the «M» by an «a» (or a «Z»).

KEY WORDS: Painting Panels, XVI Century, Jesuit Iconography of the Counter-Reformation, Engraver Giovanni Battista Cavallieri.

Recibido: 17 de abril de 2013 / Aceptado: 2 de mayo de 2013.

El objetivo de este breve artículo es dar a conocer tres importantes tablas con pinturas del siglo XVI que Ilamaron nuestra atención hace unos años cuando visitábamos el Colegio de Lecároz, situado en el navarro valle de Baztán. Las tres tablas, que han permanecido inéditas hasta la fecha, se guardaban en una sala a modo de pequeño museo, junto a un conjunto importante de pinturas del siglo

* GARCÍA GAINZA, María Concepción: "Sobre tres tablas pintadas del siglo XVI», Boletín de Arte, n. 34, Departamento de Historia del Arte, Universidad de Málaga, 2013, pp. 125-134, ISSN: 0211-8483. 
XVIII, obras enviadas por los becarios de la Real Academia de San Fernando en Roma que son copia de cuadros de pintores italianos famosos'1. Un recibo conservado en el archivo del colegio de Lecároz ofrece una relación detallada de las obras enviadas en 1891 desde la Academia al colegio de misioneros de Lecároz a petición de su superior el P. Joaquín María de Llevaneras, procurador general de misiones y ministro provincial de Castilla. En la mencionada relación no figuran las tres tablas de pintura del siglo XVI aunque su origen es claramente foráneo, ya que no se relacionan ni con la pintura navarra ni con la regional próxima. Cabe la posibilidad de que las tablas fueran donadas a raíz de las peticiones del P. Llevaneras por otro legatario o que se incorporaran a las pinturas reunidas en el Colegio con posterioridad ${ }^{2}$. El archivo convenientemente revisado no arroja luz alguna sobre su procedencia ni origen ${ }^{3}$.

Las tres tablas pintadas son de gran tamaño, cerca de dos metros de altura, presentan las tres las mismas dimensiones, lo que hace pensar que formaban parte de un conjunto, quizá un retablo. A esta idea contribuye el hecho de que las pinturas por su uniformidad formal parezcan de la misma mano, probablemente debidas al mismo pintor. El estilo de las pinturas es el propio que está vigente en algunas escuelas pictóricas hispánicas hacia 1590 ya dentro de la pintura de la Reforma católica. El anónimo pintor imprime su monograma . F M ., la F surmontada por una o y la M ligada a la T y surmontada por una A, en la tabla de San Julián repartiendo la limosna, letras que parecen de grabador y que quizás puedan corresponder a Fernando o Francisco Mata. A pesar de las búsquedas no hemos conseguido identificar la personalidad del pintor oculto bajo esas iniciales. En lo que a su iconografía respecta las tres pinturas representan la Lapidación de

1 GARCÍA GAINZA, M. ${ }^{\text {a } C o n c e p c i o ́ n, ~ " P i n t u r a s ~ d e ~ l a ~ R e a l ~ A c a d e m i a ~ e n ~ e l ~ C o l e g i o ~ d e ~ C a p u c h i n o s ~ d e ~}$ Lecároz", Academia. Boletín de la Real Academia de Bellas Artes de San Fernando, Números 94 y 95. Primer y Segundo semestre de 2002, pp. 9-22.

Estas pinturas acompañaban al famoso Cristo de Alonso Cano procedente del convento de Monserrate de Madrid que aparece citado en la relación de obras mencionadas pero sin indicar el nombre del

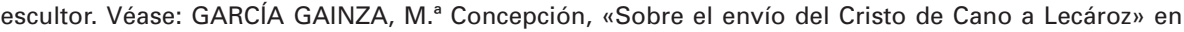
Alonso Cano y su época. Symposium internacional, Granada, 2002, pp. 151-160.

2 El conjunto de cuadros incluidas las tres tablas pintadas fueron trasladadas hace unos veinte años al convento de capuchinos de Sangüesa (Navarra) al abandonarse el Colegio de Lecároz, y allí se encuentran en la actualidad.

3 En el convento de Capuchinos Extramuros de Pamplona se halla depositado el fondo documental del Archivo de Lecároz. Su revisión no ha ofrecido ningún hallazgo documental acerca de las tres tablas. Existe únicamente una Relación de las obras de arte existentes en el convento de Padres Capuchinos de Lecároz. Lecároz, enero 1996, 25 folios, fol. 15, menciona en la sala de visitas los cuadros: $\mathrm{n}$. ${ }^{\circ} 11$. Martirio de San Esteban (tabla original). Alto: 1,75, Ancho 1,30; ${ }^{\circ}{ }^{\circ} 12$. Joven repartiendo la limosna (tabla original, estilo flamenco). Alto 1,72, Ancho 1,32; n. ${ }^{\circ} 13$. Obispo ¿exorcizando? (tabla original con muchas personas). Alto 1,80, Ancho 1,30.

Según el P. Tarsicio de Azcona a quien agradezco vivamente la revisión documental el autor de la relación fue el P. Cabodevilla. 
San Esteban, San Julián y Santa Basilisa repartiendo la limosna y santo obispo bendiciendo los campos.

\section{La Lapidación de San Esteban}

La Lapidación de San Esteban (1,75 x 1,30) muestra el martirio del protomártir según narran los Hechos de los Apóstoles, 7: 54-60. "Sacándole fuera de la ciudad le apedreaban [...]; y mientras le apedreaban, Esteban [...] gritó con fuerte voz: Señor no les imputes este pecado".

La tabla muestra al santo arrodillado sobre las dos rodillas con su cuerpo torsionado al frente y con los brazos en cruz y las manos abiertas [1]. Su cabeza y su mirada se dirigen al cielo mientras recibe la lluvia de piedras de tres verdugos que hacen círculo en torno al santo, uno de espaldas atléticas a un lado, otro que arroja la piedra con los brazos elevados y las manos puntas de frente al otro y finalmente el tercero igualmente de frente a espaldas del santo. En segundo plano se advierte un sayón más con la piedra en la mano y otros personajes, uno de los cuales carga a la espalda un cesto de piedras. En el ángulo inferior aparece un joven de espaldas desnudas y musculosos brazos que recoge las piedras del suelo en una cesta. Se representa aquí el momento milagroso del martirio en el que San Esteban imploró el perdón de sus verdugos y vio los cielos abiertos con el Padre Eterno y Jesucristo Resucitado como narran los Hechos: «Pero él, Ileno del Espíritu Santo, con los ojos fijos en el cielo, vio la gloria de Dios y a Jesús de pie a la derecha de Dios» entre nubes. La escena tiene lugar a las afueras de una ciudad de la que se ven una torre y la muralla con una ventana enrejada que parece la cárcel.

La composición de esta pintura descrita nada tiene que ver con el célebre Martirio de San Esteban del Museo del Prado de Juan de Juanes, que muestra al diácono mártir de perfil implorando con las manos juntas y los verdugos apedreándole por la espalda ${ }^{4}$, ya que sigue, como veremos, un modelo italiano.

La propagación del martirio de San Esteban tuvo lugar a través de los sermones de San Agustín y el protomártir gozó de gran devoción en Europa incluida España, donde existen varias iglesias con la advocación de San Esteban. La iconografía de la lapidación de San Esteban se difundió por medio de estampas grabadas propias de la iconografía jesuita de la Contrarreforma. En concreto, en la pintura que comentamos la composición está tomada de una estampa de la

4 ALBI, José, Joan de Joanes y su círculo artístico, Institución Alfonso el Magnánimo, Valencia, 1979, Tomo II, pp. 142-181 y Tomo III, Lámina CLXIX. 


E artículos María Concepción García Gainza

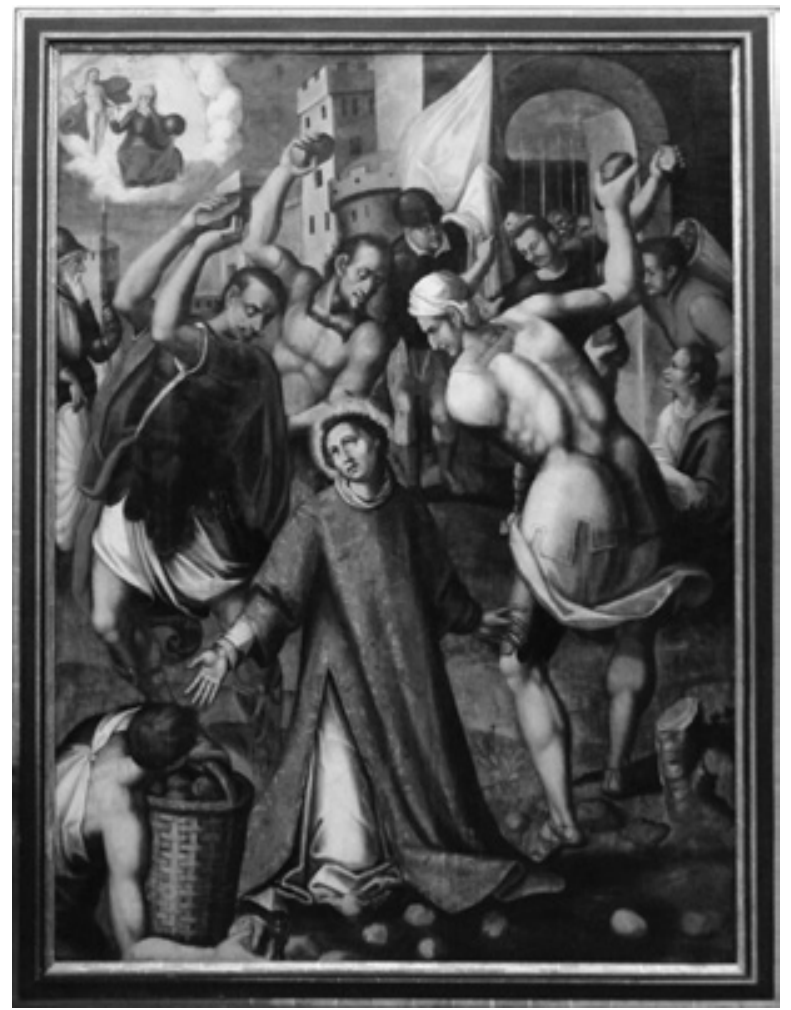

1. Lapidación de San Esteban. Convento de Capuchinos. Sangüesa (Navarra)

obra Ecclesiae Militantis Triumphi [4], impresa en Roma en 1583 y reimpresa en 1585, cuyo autor es Pomerancio, una serie impresa de treinta y una estampas en las que Giovanni Battista Cavalieri grabó escenas de martirio tomadas de los frescos del deambulatorio de la iglesia de Santo Stefano Rotondo de Roma ejecutados por el pintor Nicolo Circignani ${ }^{5}$. Esta obra sufragada por los jesuitas fue promovida por el Colegio Germano-Húngaro con el Privilegio del papa Gregorio XIII, con objeto de establecer la relación entre los primeros mártires del cristianismo y la doctrina teológica establecida en el Concilio de Trento. La obra estaba destinada a los jesuitas misioneros en los países del norte protestante por lo que fue impresa en varios países con posterioridad.

5 KIRSTIN NOREEN, Ecclesiae militantes triumphi: Jesuit Iconography and the Counter-Reformatión, The Sixteenth Century Journal, Vol. 29, No. 3 (Autumn), 1998, pp. 689-715.

TSOUMIS, K., Giovanni Battista Cavallieri's Ecclesiae militantes triunphi: Jesuits, Martyrs, Print, and the Counter-Reformation, Heritage Branch, Ottawa, 2005, pp. 1-126. 
2. Lapidación de San Esteban. Giovanni Battista

Cavallieri: Ecclesiae militantis triumphi (1585)

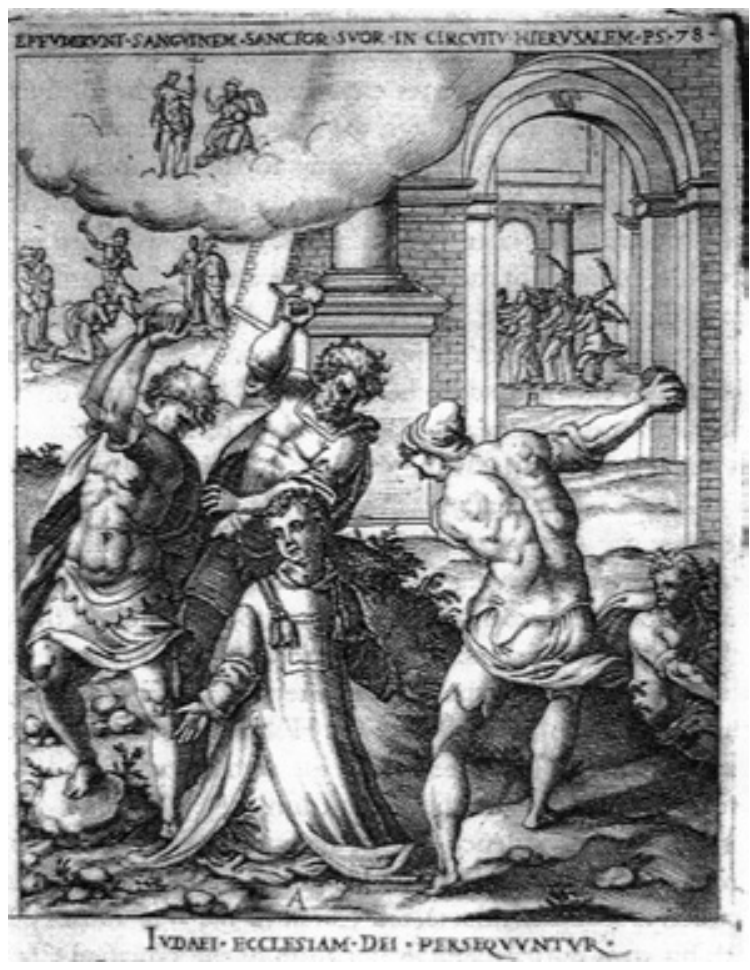

El pintor de la Lapidación de San Esteban tomó de este grabado ${ }^{6}$ las cuatro figuras principales, el santo y tres sayones y desestimó las escenas secundarias de los apóstoles perseguidos y Santiago muerto a espada [2].

La copia literal de la composición es evidente en la figura de San Esteban arrodillado con los brazos y manos abiertas como Cristo en la cruz y la dalmática abierta en su rodilla con el alba plegada en el suelo con parecidos pliegues. El verdugo de espaldas reproduce la exagerada musculatura del grabado y su brazo extendido hacia fuera con la piedra y el de frente eleva los dos brazos hacia

6 Eclesiae Militantis Triunphi (Roma, 1585), 31 estampas, $n .^{\circ} 2$. El grabado lleva en la parte superior la inscripción: EFFVDERVNT SANGVINEM SANCTORVM TVORVM HIERVSALEN, ps. 78, que corresponde a la Gradual de Inocentes. En la parte inferior:

IVDAEI. ECCLESIAM.DEI.PERSEQVVNTVR.
A.STEPHANVS lapidatur
B. APOSTOLI caeduntur
C. IACOBVS frater loannis occiditur gladio

que identifican la escena principal de la Lapidación de San Esteban y las secundarias del fondo de los Apóstoles perseguidos y Santigo hermano de Juan muerto a espada.

Biblioteca Angelica. Roma, c. 6.26. 


\section{- artículos María Concepción García Gainza}

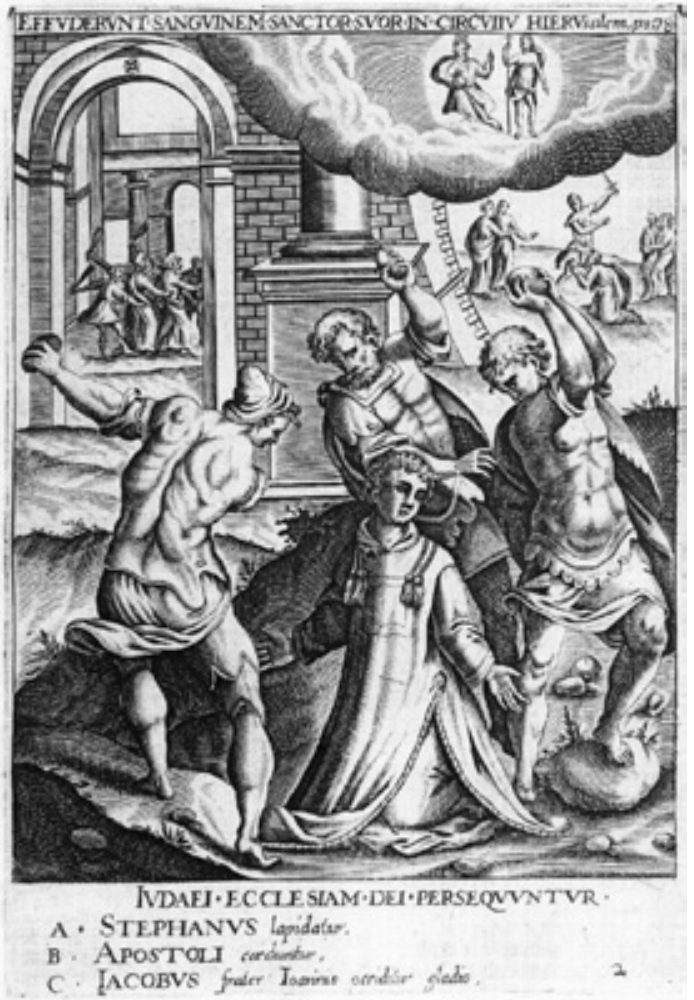

3. Lapidación de San

Esteban. Jan van Haelbeck

arriba para arrojar la piedra con las manos; en ambos la disposición de las piernas es idéntica, incluso su modelado.

Igualmente el verdugo de espalda levanta un solo brazo hacia arriba como el grabado e igual fidelidad puede verse en el Padre Eterno sedente y en el Cristo resucitado. Las figuras restantes de la pintura de la tabla son añadidas por el pintor a excepción del muchacho con la cesta de piedras que ha sido trasladado al ángulo de la tabla y asimismo el fondo arquitectónico clásico ha sido transformado en la tabla en una muralla, si bien mantiene el arco triunfal a la derecha.

Un grabado idéntico, perteneciente al ejemplar de Ecclesiae Militantis Triunphi publicada en París por Jean Leclerc IV7, reproduce el grabado romano

7 Museo Británico. Departamento: Grabados y Dibujos, n. ${ }^{\circ} 18630509.763$. Publicado por Jean Leclerc IV IFF 227-258.

Sobre Jean Leclerc (1586-1633) impresor francés, véase PRÉAVD, Maxime, CASELLE, Pierre, GRIVEL, Marianne, LE BITOUZÉ, Corinne, Dictionnaire des éditeurs d'estampes à Paris sous l'Ancien Régime, Promodis, 1987, pp. 210-211. 
4. Giovanni Battista Cavallieri Ecclesiae militantis triumphi (1585). Frontispicio

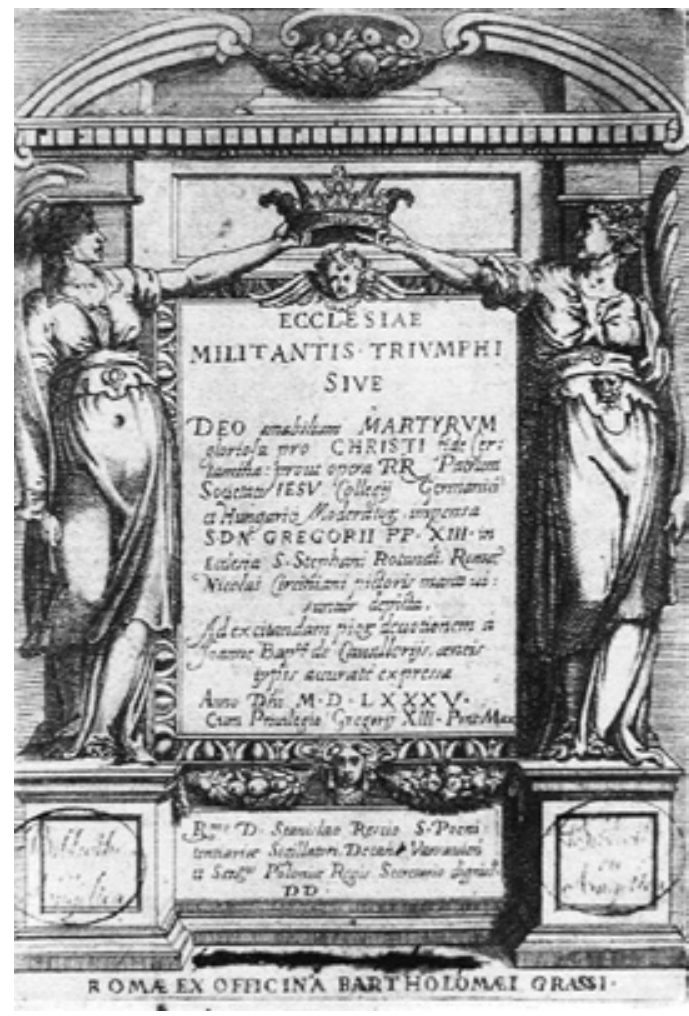

invertido lateralmente y es obra del grabador flamenco Jan van Haelbeck que trabajó en París entre 1600 y 1630 [3].

La fecha del grabado de Cavallieri permite situar la pintura con posterioridad a 1585, y su ejecución y colorido manierista a base de malvas y amarillos la definen tanto por su forma como por su contenido como una característica manifestación de pintura de la Contrarreforma.

\section{San Julián y Santa Basilisa repartiendo la limosna}

Por su rareza iconográfica este tema resulta difícil de identificar pero todos los detalles de la pintura contribuyen a ello [5]. San Julián, perteneciente a una familia noble, contrajo matrimonio con Santa Basilisa pero ambos decidieron Ilevar una vida de completa castidad. Tiempo más tarde pasó San Julián a integrarse 


\section{Q: artículos}
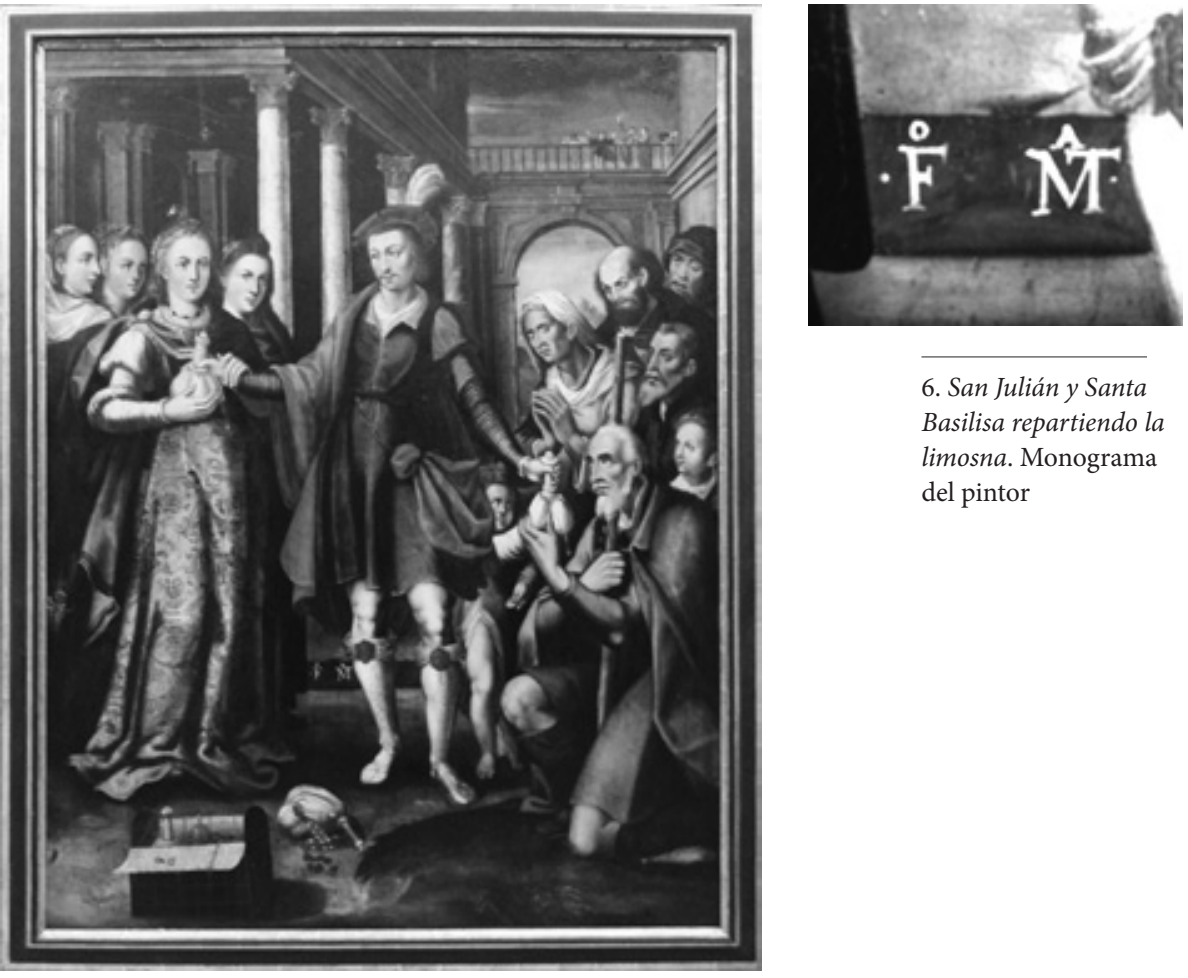

6. San Julián y Santa Basilisa repartiendo la limosna. Monograma del pintor

5. San Julián y Santa Basilisa repartiendo la limosna. Convento de Capuchinos. Sangüesa (Navarra)

en una comunidad de la que fue un santo abad y Santa Basilisa formó con otras vírgenes un monasterio próximo al de su esposo del que también fue abadesa. Ambos recibieron el martirio en Antioquía en tiempos del emperador Diocleciano, en el siglo IV.

Considerados como modelo de matrimonio cristiano, en la pintura de la tabla aparecen ambos ricamente vestidos de boda en el momento que han decidido después de su matrimonio entregar sus riquezas y la dote de la esposa a los pobres para seguir después su propio camino. San Julián ocupa el centro

8 La iconografía de San Julián y Santa Basilisa no es muy conocida y con frecuencia se confunde con la de San Julián el Hospitalero mucho más representada. Para la vida de San Julián y Santa Basilisa consultar Año Cristiano, Tomo I, enero-marzo, Biblioteca de Autores Cristianos, Madrid 1959, 9 de enero, cuyo autor es Valentín Soria. Agradecemos al profesor Ricardo Fernández Gracia su valiosa ayuda para la identificación de esta iconografía. 
de la composición y viste túnica corta, manto, calzas con broches y se toca con sombrero de plumas y Santa Basilisa, a quien acompañan tres elegantes donceIlas, lleva vestido de brocado en oro y manto azul. El arca matrimonial con las joyas aparece abierta en el suelo con las dos alianzas sobre la tapa que aluden al abandono de la vida conyugal para retirarse a la oración. El santo toma bolsas de oro que le entrega Santa Basilisa a la vez que esta coge con afecto su mano y San Julián entrega con la otra mano las riquezas a los pobres que se hallan a su lado.

Entre estos se distingue un anciano arrodillado que recibe la bolsa de oro acompañado de una anciana, dos niños y tres mendigos más, uno de ellos con un bastón.

La escena tiene lugar en un palacio según sugiere una arquitectura clásica de columnas de mármol, arco de triunfo y balaustrada sobre la que se abren las lejanías. Entre las figuras de San Julián y Santa Basilisa, sobre fondo oscuro, resaltan las letras .F M., la F surmontada por una o y $\mathrm{M}$ ligada a una $\mathrm{T}$ y surmontada por una A, que corresponderán al anónimo pintor [6].

\section{Santo obispo bendiciendo los campos}

Se representa en el centro de la composición a un santo obispo revestido de pontifical bendiciendo con el hisopo ante un altar que preside un crucifijo y un libro abierto sobre el que apoya su mano entre dos velas [7]. Sirve de fondo al altar una gran parra arrancada cuajada de pámpanos que cabe la duda si puede deberse a la acción milagrosa de la bendición episcopal. Rodean al obispo un grupo de eclesiásticos pertenecientes a distintas iglesias o pueblos pues portan dos cruces procesionales con sus correspondientes mangas. Los eclesiásticos se representan en diversas actitudes, unos siguen la bendición con sus breviarios, otros sostienen las cruces o el báculo del obispo y uno permanece en primer término arrodillado. Algunos cubren sus cabezas con bonetes y uno lleva anteojos.

Cierra el círculo en primer término un hombre arrodillado y dos mujeres sentadas de espalda, una de ellas con un niño desnudo en brazos y otro niño de espaldas presenciando la escena.

Sirve de nexo de ambos grupos el retrato de un hombre arrodillado y con las manos juntas. Situado en el margen de la tabla dirige su mirada fijamente al espectador. Se trata de un hombre de cierta edad a juzgar por su barba canosa y cierta posición social por su indumentaria de cuello y puños blancos según la moda de la época de Felipe II. Posiblemente se tratará del donante. 


\section{- artículos María Concepción García Gainza}

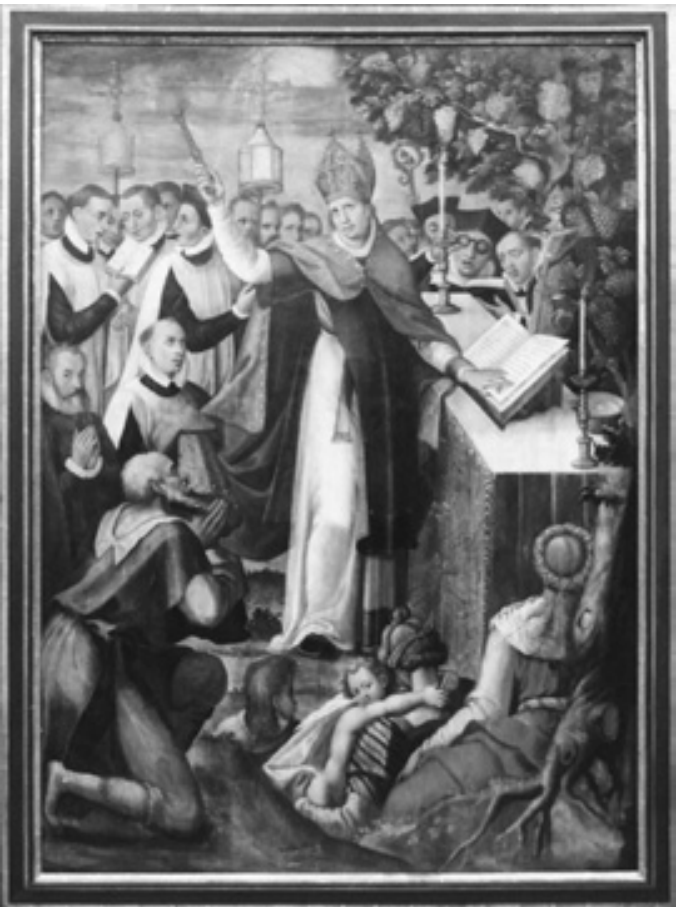

7. Santo obispo bendiciendo los campos. Convento de Capuchinos. Sangüesa (Navarra)

No resulta fácil identificar con precisión de qué escena se trata y tampoco quién es el santo obispo que bendice. Cabría la posibilidad de que se tratase de San Vicente de Zaragoza, diácono y mártir, que era patrón de los viticultores a quienes enseñó el cultivo de la vid, la poda y la vendimia ${ }^{9}$. Esta sería la razón de la presencia de la gran parra cuajada de racimos que aparece en la pintura como también su tronco seco y podado del primer término. La dificultad para esta identificación estriba en que San Vicente no fue obispo, si bien como relata Leyenda Dorada ${ }^{10}$, dada su facilidad de palabra y elocuencia, el obispo Valerio delegó en él todo lo relacionado con la predicación y la enseñanza en aquella diócesis. Pese a ello resulta difícil aceptar que aparezca revestido de obispo aunque como puede apreciarse lleva la dalmática de diácono debajo de la capa pluvial ${ }^{11}$.

9 REAU, L., Iconografía del Arte Cristiano Iconografía de los santos, P. Z, Tomo 2, vol. 5, Ediciones del Serbal, Barcelona, 1998, pp. 322-328.

10 VORAGINE DE LA, S., La Leyenda Dorada, Alianza Forma, Madrid, 1992, pp. 120-123.

11 Conocemos el caso de una tabla del siglo XV, obra de F. Comes perteneciente al retablo de Santa María de Gracia, ahora en Museo de Palma de Mallorca, en la que se representaba a San Vicente como un santo obispo. Al parecer tras una restauración ha quedado convertido en diácono, MATEO IBARS, M. de los D., Iconografía de San Vicente Mártir, Tomo I, Pintura, Institución Alfonso el Magnánimo, Valencia, 1980, pp. 58-59, Láms. 11.1-11.6. 